\title{
Comparative Study of the Influence of Helium and Argon Plasma on Crystallogenic Properties of the Blood
}

\author{
Andrew K. Martusevich, ${ }^{a, b, *}$ Alexander G. Galka, ${ }^{a, c}$ Elena S. Golygina, ${ }^{a}$ \\ Alexandra S. Fedotova, ${ }^{a, b}$ Alexander N. Tuzhilkin, ${ }^{a, b}$ \& \\ Svetlana L. Malinovskaya ${ }^{a}$ \\ aPrivolzhsky Research Medical University, Nizhny Novgorod, Russia; ' $N$ Nizhny Novgorod \\ State Agricultural Academy, Nizhny Novgorod, Russia; 'Institute of Applied Physics, Russian \\ Academy of Sciences, Nizhny Novgorod, Russia \\ *Address all correspondence to: Andrew K. Martusevich, Privolzhsky Research Medical University, 10/1 Minin Square, \\ Nizhny Novgorod 603950, Russia; Tel.: +7-909-144-91-82, E-mail: cryst-mart@yandex.ru
}

\begin{abstract}
The aim of this study was to compare the biological effects of helium and argon plasma in vitro. The cold plasma was generated using a device developed at the Institute of Applied Physics of the Russian Academy of Sciences (Nizhny Novgorod), which uses the principle of microwave ionization of the gas flow. We studied the crystallogenic activity of blood plasma. The description of dehydrated blood plasma samples was performed morphologically, using a system of visuametric parameters. Main morphometric parameters for analysis of blood plasma crystallization include crystallizability, structure index, facia destruction degree, and size of marginal zone. This study allowed us to establish that the effect of helium and argon in both free and ionized forms on the crystallogenic activity of blood varies significantly. The effect of cold plasma in general should be described as more favorable relative to the corresponding nonionized fluxes. At the same time, the most optimal nature of the reaction of the biological fluid was recorded when using a helium cold plasma. A specific feature of the helium flow is the suppression of the crystallogenic activity of the biological fluid, and the argon flow leads to its increase. Ionization of gases optimizes the nature of their influence, moreover, according to the modeling effect, the helium cold plasma is closest to the control sample.
\end{abstract}

KEY WORDS: cold plasma, crystallization, gas carriers, biocrystallomics

\section{INTRODUCTION}

Cold plasma is known to have numerous physical and biological effects, the significance of which is currently only beginning to be revealed..$^{1-3}$ This is evidenced by the rapid increase in the number of publications devoted to this topic, and they relate not only to medicine, ${ }^{1,3}$ but also, for example, to agriculture, ${ }^{4}$ crop production, ${ }^{5-7}$ and the food industry. ${ }^{4,8,9}$ At the same time, the main focus of these studies, regardless of the subject, is based on the application of various aspects of the antibacterial activity of the factor under consideration. ${ }^{1,8-11} \mathrm{It}$, in turn, is associated with initiating the formation of reactive oxygen and nitrogen species by cold plasma. ${ }^{2,12,13}$

No less important is the question of the optimal choice of a carrier gas for a cold plasma, which is proposed by various authors as atmospheric air, helium, or 
argon..$^{3,12,14-16}$ In the literature, there is even information about the possibility of using a polycomponent nitrogen-argon, ${ }^{17}$ helium-argon, ${ }^{12}$ and argon-air/argon-oxygen ${ }^{18}$ plasma. On the other hand, the influence of the plasma carrier gas on its biological effects, as well as the features of air, helium, and argon plasma, is not well known, which makes it difficult to answer this question. A priori, it can be assumed that the nature of the ionized particles in the flame stream has a certain significance in the implementation of its action on biological objects, ${ }^{2,8,13,19,20}$ and this circumstance is extremely important for the correct selection of the carrier gas for a specific clinical or experimental tasks.

In a previously published study, we showed that helium cold plasma significantly transforms the crystallogenesis of blood serum in vitro, which is additionally accompanied by a change in its dielectric properties. ${ }^{26}$ In addition, we have previously demonstrated the dependence of metabolic shifts in the biological fluid on the time of exposure to cold plasma. ${ }^{21}$ At the same time, the effect of the carrier gas on the modulating effect of cold plasma on the crystallization of blood serum has not been studied. Based on this, the aim of this study was to compare the biological effects of helium and argon plasma in vitro (for example, the effect on the crystallogenic properties of blood).

\section{MATERIAL AND METHODS}

\section{A. Material and Experiments Description}

The study of the biological effects of cold plasma using various carrier gases was carried out on a stand previously described by the experiment. ${ }^{26}$ The effect was carried out by direct contact of the plasma torch with the surface of the biological fluid contained in a plastic tube (the distance between them is 2 to $3 \mathrm{~mm}$ ). Helium and argon grade A (99.9\% purity) were used as carrier gases. The study material was whole blood of healthy people $(n=12)$, and each sample was divided into five portions. The first of them was a control (without manipulation); the second was treated with a stream of nonionized helium the third was treated with helium cold plasma; the fourth one was treated with nonionized argon; and the fifth was treated with argon cold plasma. The cold plasma was generated using a device developed at the Institute of Applied Physics of the Russian Academy of Sciences (Nizhny Novgorod), which uses the principle of microwave ionization of the gas flow, previously described by us. ${ }^{21}$ The temperature of the weakly ionized gas jet was $32^{\circ} \mathrm{C}$, and the average power of the source did not exceed $20 \mathrm{~W}$. The distance from the end of the plasma torch to the surface of the biological fluid was 1.0 to $1.5 \mathrm{~cm}$. The duration of the treatment was 1 minute, and the exposure after its completion was 15 minutes. In order to study the potential participation of the thermal factor in the implementation of the effects of cold plasma and to correctly calculate the crystalloscopic parameters of the biological medium, the temperature of the blood sample was estimated using a portable infrared thermometer (Fluke $59 \mathrm{Max}+$ ). 


\section{B. Preparation of Blood Samples}

After exposure, the blood samples were centrifuged according to the standard procedure (1500 rpm, $20 \mathrm{~min}$ ), isolating the blood plasma. The resulting plasma of each sample was applied in the form of drops (volume, $50 \mu \mathrm{L}$ ) on a previously cleaned slide. After that, free (without thermal stimulation) dehydration of the drops was provided for 24 hours.

\section{Estimation of Crystallogenic Properties of Blood Samples}

To conduct a crystalloscopic study, all samples of biological fluid were centrifuged according to the standard method until plasma was obtained. Then we studied the intrinsic crystallogenic activity of blood plasma by classical crystalloscopy. ${ }^{21-23}$ The description of dehydrated blood plasma samples, called facia, was performed morphologically and using a system of visuametric (visuametry is a method of morphometrically describing crystallization effects with visual noninstrumental criteria) parameters that characterize the qualitative and quantitative aspects of the process of crystallization of the biological medium. Main morphometric parameters for analysis of blood plasma crystallization include crystallizability, structure index, facia destruction degree, and size of marginal zone. ${ }^{21-23}$ All these parameters are calculated in direct four-points scale. Crystallizability is characterizing the density of crystals in dried specimens (defined as less than 10;10 to $20 ; 20$ to 30 , and more than 30 crystal structures in the field of view for $0,1,2$, and 3 points, respectively). Structure index is used to estimate the complexity of the resulting crystal elements. The presence of only amorphous structures corresponds to 0 points of structure index, the presence of single crystals is 1 point, the combination of single crystals and dendrites is 2 points, and the formation of numerous dendritic elements is 3 points, respectively. Facia destruction degree is an integral indicator that characterizes the "correctness" of the structure formation of crystal elements. When crystals of an ideal shape form, which do not have any signs of destruction, 0 points are fixed. The presence of minimal signs of crystal destruction without a significant violation of their shape is treated as 1 point. Significant destruction of crystals with the possibility of unambiguous determination of their shape is estimated as 2 points. Total destruction of crystalline elements in a dried sample without the possibility of distinguishing their shape is regarded as 3 points. The size of marginal zone describes the size of the marginal protein belt of the facias. If this belt is completely or almost completely absent, the parameter value is 0 points. The minimum severity of this zone (no more than $1 / 5$ of the facias radius) corresponds to 1 point. A wide marginal belt (1/5 to $1 / 3$ of the facias radius) is considered 2 points, and the maximum wide one (more than $1 / 3$ of the radius) is 3 points.

\section{Ethic Approval}

This study was approved by Local Ethic Committee of Privolzhsky Research Medical University (04/2018).

Volume 11, Issue 1, 2021 


\section{E. Statistics}

The obtained data was processed statistically in the software package Statistica 6.1 for Windows. The normality of the distribution of parameter values was evaluated using the Shapiro-Wilk test. Accounting for the nature of the attribute distribution, the KraskalWallace $H$ test was used to assess the statistical significance of differences.

\section{RESULTS}

Based on the conducted studies, the studied physical factors specifically transformed the crystalloscopic picture of the biological fluid. The example shown in Fig. 1A shows that the blood plasma facias of an intact sample have a uniform texture and relatively regular
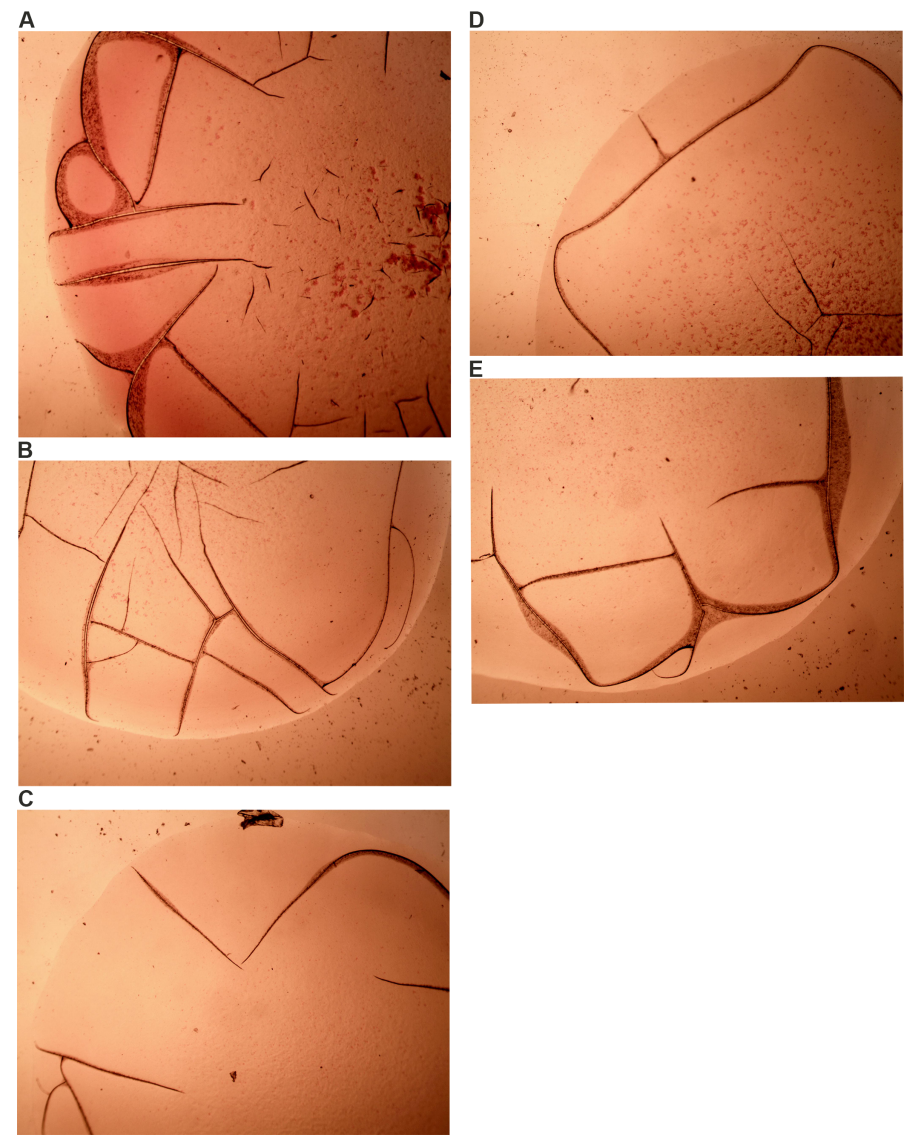

FIG. 1: Crystalloscopic facias of blood plasma in control (without any manipulations) after 1-minute treatment (light microscopy; $\times 60$ ): (A) control (intact) blood specimen; (B) blood processing with helium flow; (C) blood processing with helium cold plasma; (D) blood processing with argon flow; (E) blood processing with argon cold plasma 
radial faults and fairly well-defined zones. A wide marginal zone is especially clear in the micropreparation. In addition, a small number of single crystal elements and small cracks, including three-point beam ones, are found in the facias.

Examples of crystallograms in the treatment of blood with ionized and nonionized helium and argon streams are shown in Fig. 1B-1E. It was found that the features of the crystallization of blood plasma pretreated with helium flow, compared with the control sample, are a moderate decrease in the density of crystal structures, chaotic faults present in all facias zones, and a narrowing of the edge belt of the micropreparation (see Fig. 1B). This indirectly indicates the negative influence of this factor on the physical and chemical properties of the biological fluid.

In contrast, the use of nonionized argon flow yields increased density of single crystals in facias blood plasma without their complications (no dendritic structures), reducing the number of faults (the formation of a single girdling faults), and significant reduction in the size of the boundary zone (see Fig. 1D). All this points to significant negative changes in the physical and chemical parameters of the biological fluid, including the destabilization of its proteome.

A fundamentally different character of structurization was found for cold plasma, and there are features of specificity due to the carrier gas used. Thus, the most optimal variant of facias was observed when blood was treated with cold helium plasma (see Fig. 1C). In this case, the density of the structural elements represented exclusively by single crystals was comparable to the control sample. In addition, in these facias, the degree of destruction of the elements was moderate, and the marginal protein zone was well defined but comparable to blood samples treated with nonionized helium.

Significant features were found in dried blood plasma droplets exposed to argon cold plasma (see Fig. 1E). They showed complicated structural elements of the facias (the appearance of a moderate number of dendritic crystals) but maintained their density. At the same time, the degree of destruction of the elements was significantly lower both in comparison with the control sample and the facias of blood plasma treated with nonionized argon. In addition, the use of argon cold plasma contributed to the expansion of the marginal protein belt in dried drops of biological fluid.

Based on the presented pictures, it can be concluded that all the considered effects specifically transform the crystallogenic activity of blood plasma.

The data of the morphological analysis of the crystallograms are fully consistent with the results of their criterion evaluation. Thus, treatment of blood samples with nonionized helium and argon significantly changes the level of the main quantitative indicator, crystallizability, which reflects the density of the distribution of structural elements over the facias texture (Fig. 2). Interestingly, helium has a moderate inhibitory effect (1.19 times; $p<0.05$ ), whereas argon exposure significantly increases crystallizability $(1.58$ times; $p<0.05)$. The ionization of each of the considered gas flows significantly transforms the nature of their influence on the crystallogenic activity of the biological fluid. In particular, the use of helium cold plasma, in contrast to nonionized helium, retains the crystallizability value at the level characteristic of the control sample, which was not manipulated in any way. Argon ionization also shows that the parameter

Volume 11, Issue 1, 2021 


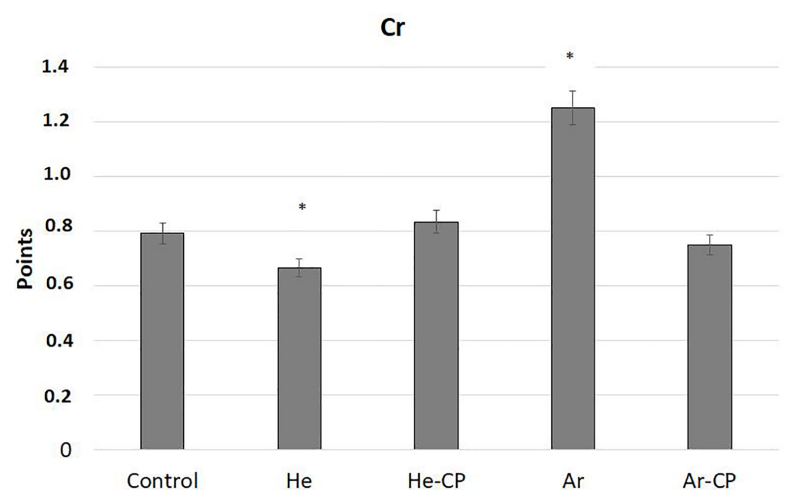

FIG. 2: Crystallizability (Cr, points) in facias of human blood plasma in control and after 1-minute treatment with different exposures (He, helium; He-CP, helium cold plasma; Ar, argon; Ar-CP, argon cold plasma) (*statistical significance of differences relative to the control sample, $p<0.05$ ). The values of the parameters were calculated by visual morphometry of dried samples of biological liquid.

approaches physiological values, while remaining statistically indistinguishable from the control. At the same time, the parameter level is lower than when using helium plasma (by $11.1 \%$; $p<0.05$ ).

Such a variability study of the influence of gas flow is observed in the structure index that reflects the complexity of the resulting crystal structures (Fig. 3). After treatment of blood for 1 minute with helium in nonionized form, the level of the structure index is significantly reduced compared with the control sample $(-39.2 \% ; p<0.05)$, indicating the inhibition of crystallization under the action of this factor. This is fully

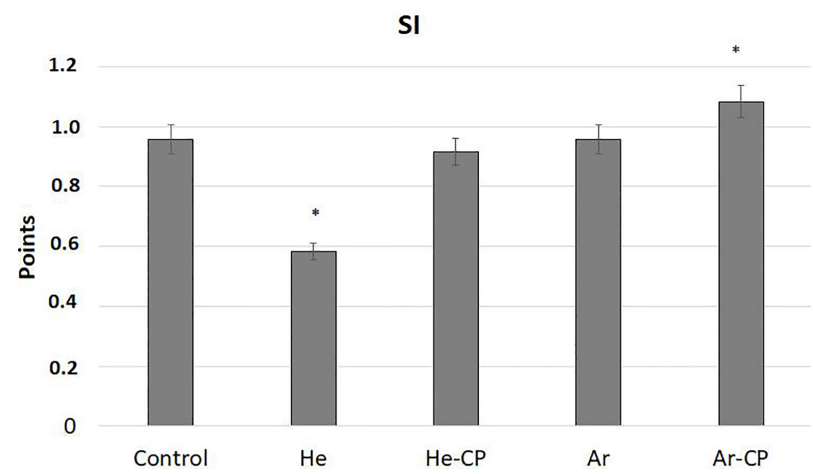

FIG. 3: Structure index (SI, points) in facias of human blood plasma in control and after 1-minute treatment with different exposures (He, helium; He-CP, helium cold plasma; Ar, argon; Ar-CP, argon cold plasma) (*statistical significance of differences relative to the control sample, $p<$ 0.05 ). The values of the parameters were calculated by visual morphometry of dried samples of biological liquid. 
consistent with the change in crystallizability relative to the control. On the contrary, when using nonionized argon, the structure index does not change, which (in combination with the dynamics of crystallizability) indicates only quantitative rearrangements of the crystallogenic properties of the biological fluid under this influence.

Ionization of gas flows, as in the case of crystallizability, has a significant effect on the nature of shifts in the structure index (see Fig. 3). Thus, the cold helium plasma does not cause a significant change in the parameter value, wheras the argon plasma contributes to a moderate increase in its value $(13.0 \% ; p<0.05$ relative to the control sample). This further characterizes the specificity of the influence of the factors under consideration. At the same time, the use of helium plasma eliminates the inhibitory effect recorded when using nonionized helium. On the contrary, argon ionization takes the structure index out of the physiological range.

An important parameter that integrally characterizes the crystallogenic activity of a biological fluid is the facia destruction degree (Fig. 4). It was found that both nonionized gas flows significantly and almost equally lead to an increase in the level of the parameter (by 59.5\% and 51.4\% for helium and argon, respectively; $p<0.05$ for both cases). This indicates the negative nature of the influence of both factors on the crystallogenic properties of blood plasma and, consequently, its physical and chemical parameters and component composition. Ionization of gases significantly transforms the effect of their action on the destruction degree of plasma facias. In particular, when using helium cold plasma, the indicator becomes indistinguishable from the level of the control sample, whereas when treating blood with argon cold plasma, an extremely pronounced decrease in the parameter value was noted (by 2.32 times relative to the sample with which no manipulations were performed; $p<0.05$ ). This indicates the optimal effect of the helium cold plasma on the biological object under consideration.

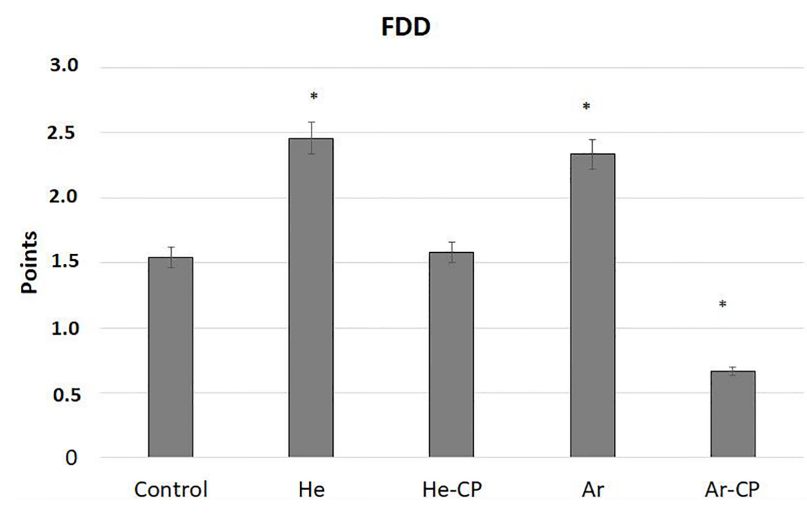

FIG. 4: Facia destruction degree (FDD, points) in facias of human blood plasma in control and after 1-minute treatment with different exposures (He, helium; He-CP, helium cold plasma; Ar, argon; Ar-CP, argon cold plasma) (*statistical significance of differences relative to the control sample, $p<0.05)$. The values of the parameters were calculated by visual morphometry of dried samples of biological liquid.

Volume 11, Issue 1, 2021 
Finally, the fourth main evaluation parameter (the size of marginal zone of biological fluid facia) shows the extent and condition of the protein component of the biological fluid. It also recorded substantial variation in the studied impacts (Fig. 5). Helium and argon in nonionized form moderately reduced the number, and severity of this effect is higher in argon ( $-13.7 \%$ versus $-27.3 \%$, respectively; $p<0.05$ for both factors). This indicates a greater optimality of the effect of helium on the blood proteome. Helium cold plasma did not change the size of the marginal zone of blood plasma facias relative to nonionized helium. At the same time, the parameter level in this case remained moderately lower than that detected for control samples $(p<0.05)$. This fact indirectly indicates an insignificant effect of the helium plasma on the protein component of the biological fluid.

On the contrary, the use of argon cold plasma in the treatment significantly expanded the size of the marginal belt of blood plasma facias (see Fig. 4), and the value of the corresponding indicator increased significantly in comparison with argon in the nonionized form (by 1.56 times; $p<0.05$ ). It should be noted that the parameter value in these samples is also higher relative to the control (by 13.7\%; $p<0.05$ ). This indicates a distinct rearrangement of the blood plasma proteome under the action of argon cold plasma.

\section{DISCUSSION}

The problem of choosing the optimal carrier gas for cold plasma is currently quite acute. The possibility and prospects of using atmospheric air, helium, and argon for this purpose are actively discussed in the literature. ${ }^{2-4,15,19}$ At the same time, each of them potentially has its own characteristics and can be useful for specific cases. Meanwhile, a

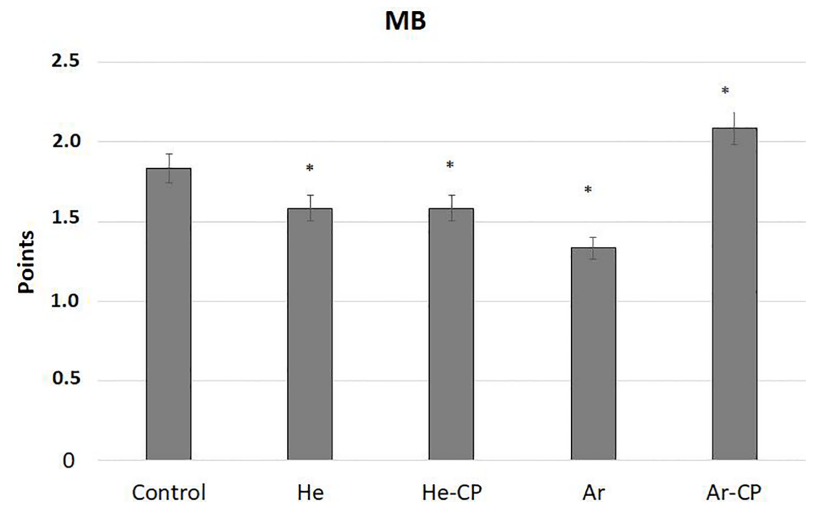

FIG. 5: Size of marginal zone (MB, points) in facias of human blood plasma in control and after 1-minute treatment with different exposures (He, helium; He-CP, helium cold plasma; Ar, argon; Ar-CP, argon cold plasma) (*statistical significance of differences relative to the control sample, $p<0.05)$. The values of the parameters were calculated by visual morphometry of dried samples of biological liquid. 
comparative analysis of the biological effects of individual cold plasma carrier gases is still present only in a few studies. ${ }^{12,15}$ Therefore, in our study, the task was set to compare their actions on a simple model biological object - human whole blood samples - in in vitro experiments.

According to the morphological analysis of facias and its morphometric assessment, blood treatment with nonionized helium flow has an adverse effect on the nature of dehydration structuring of the biological fluid. This is manifested in a significant inhibition of its crystallogenesis both in quantitative (crystallizability) and qualitative (structure index) indicators. In addition, in this case, there was a significant increase in the severity of destructive changes in the crystal structures of the facias, as well as a decrease in the size of the marginal zone is concentrating the protein component of the biological fluid. ${ }^{22-24}$ As shown by a number of studies, the effect of gas flows and cold plasma on biological objects (in particular, isolated cells) is associated with increased generation of reactive oxygen and nitrogen species. ${ }^{12,13,18,20,25}$ In our previous experiments using ironinduced biochemiluminescence, the severity of free radical processes and the state of the antioxidant system directly depended on the exposure time to cold plasma. ${ }^{21}$ Taking this into account, we assume that under the used mode of application of the helium flow, oxidative stress is realized in the blood samples, which leads to the detected shifts in their crystallogenic properties. ${ }^{26}$

A similar negative effect was observed for nonionized argon. Under the influence of this factor, there was an activation of plasma crystallogenesis associated with an increase in the density of structural elements without their complication. Such changes in dehydration structurization should be regarded as unfavorable, since they are accompanied by a pronounced increase in the degree of destruction of the facias in comparison with the control sample. In addition, the use of argon causes a significant narrowing of the edge zone, which indicates a decrease in the concentration of protein macromolecules of the native conformation and structure. ${ }^{22-24}$ These features, as in the case of blood treatment, suggest the formation of argon-induced oxidative stress in the biological fluid.

The effect of cold plasma in general should be described as more favorable relative to the corresponding nonionized fluxes. At the same time, the most optimal nature of the reaction of the biological fluid was recorded when using a helium cold plasma. Under the influence of this factor, the density of crystal elements and the complexity of their structure practically do not differ from the control sample. When treated with helium plasma, there is also no increase in the facia destruction degree, and the marginal protein zone decreases only moderately. These facts indicate that the concentration of reactive oxygen species induced by the considered exposure remains in the physiological range.

Finally, argon cold plasma demonstrates specific features of blood plasma crystallogenesis modulation. They are manifested in the formation of single dendritic structures in combination with single crystals, but their total density remains at the level of the control sample. At the same time, the facias destruction degree the under this influence is detected even at lower values than in an intact sample. In addition, in dried drops of blood plasma treated with argon cold plasma, an increased size of the marginal zone was recorded, but in this case it is represented by numerous crystallization belts. This

Volume 11, Issue 1, 2021 
circumstance, according to some authors ${ }^{24,27}$ and the results of our previous studies, $, 22,23$ can negatively characterize the influence of the factor, since it can act as an indirect sign of nitrosative stress.

Thus, the use of the crystalloscopic test made it possible to verify the specific actions of ionized and nonionized gas flows of helium and argon on the biological system (for example, human whole blood).

\section{CONCLUSIONS}

The conducted studies allowed us to establish that the effect of helium and argon in both free and ionized forms on the crystallogenic activity of blood varies significantly. At the same time, the treatment of blood with streams of nonionized helium and argon has a negative effect on the dehydration structurization of blood plasma, leading to a pronounced increase in the degree of destruction of elements and narrowing of the marginal zone of the dried specimen (facia). A specific feature of the helium flow is the suppression of the crystallogenic activity of the biological fluid, and the argon flow leads to its increase. Ionization of gases optimizes the nature of their influence, moreover, according to the modeling effect, the helium cold plasma is closest to the control sample.

\section{REFERENCES}

1. Alkawareek MY, Gorman SP, Graham WG, Gilmore BF. Potential cellular targets and antibacterial efficacy of atmospheric pressure non-thermal plasma. Int J Antimicrob Agents. 2014;43(2):154-60. doi: 10.1016/j.ijantimicag.2013.08.022.

2. Dobrynin D, Fridman D, Friedman G, Fridman A. Physical and biological mechanisms of direct plasma interaction with living tissue. New J Phys. 2009;11(11):115020. doi: 10.1088/1367-2630/11/11/115020.

3. Laroussi M. Low-temperature plasmas for medicine? IEEE Trans Plasma Sci. 2009;37:714-25. doi: 10.1109/tps.2009.2017267.

4. Scholtz V, Pazlarova J, Souskova H, Khun J, Julak J. Nonthermal plasma-a tool for decontamination and disinfection. Biotechnol Adv. 2015;33(6 Pt 2):1108-19. doi: 10.1016/j.biotechadv. 2015.01.002.

5. Feng X, Ma X, Liu H, Xie J, He C, Fan R. Argon plasma effects on maize: Pesticide degradation and quality changes. J Sci Food Agric. 2019;99(12):5491-8. doi: 10.1002/jsfa.9810.

6. Kim SY, Lee SY, Min SC. Improvement of the antioxidant activity, water solubility, and dispersion stability of prickly pear cactus fruit extracts using argon cold plasma treatment. J Food Sci. 2019;84(10):2876-82. doi: 10.1111/1750-3841.14791.

7. Sheteiwy MS, An J, Yin M, Jia X, Guan Y, He F, Hu J. Cold plasma treatment and exogenous salicylic acid priming enhances salinity tolerance of Oryza sativa seedlings. Protoplasma. 2019;256(1):79-99. doi: 10.1007/s00709-018-1279-0.

8. Guo L, Xu R, Gou L, Liu Z, Zhao Y, Liu D, Zhang L, Chen H, Kong MG. Mechanism of virus inactivation by cold atmospheric-pressure plasma and plasma-activated water. Appl Environ Microbiol. 2018;84(17):e00726-18. doi: 10.1128/AEM.00726-18.

9. Butscher D, Zimmermann D, Schuppler M, von Rohr PR. Plasma inactivation of bacterial endospores on wheat grains and polymeric model substrates in a dielectric barrier discharge. Food Control. 2016;60:636-45. doi: 10.1016/j.foodcont.2015.09.003.

10. Alshraiedeh NH, Higginbotham S, Flynn PB, Alkawareek MY, Tunney MM, Gorman SP, Graham WG, Gilmore BF. Eradication and phenotypic tolerance of Burkholderia cenocepacia biofilms 
exposed to atmospheric pressure non-thermal plasma. Int J Antimicrob Agents. 2016;47(6):446-50. doi: 10.1016/j.ijantimicag.2016.03.004.

11. Flynn PB, Busetti A, Wielogorska E. Chevallier OP, Elliott CT, Laverty G, Gorman SP, Graham WG, Gilmore BF. Non-thermal plasma exposure rapidly attenuates bacterial AHL-dependent quorum sensing and virulence. Sci Rep. 2016;6:26320. doi: 10.1038/srep26320.

12. Schweigert I, Zakrevsky D, Gugin P, Yelak E, Golubitskaya E, Troitskaya O, Koval O. Interaction of cold atmospheric argon and helium plasma jets with bio-target with grounded substrate beneath. Appl Sci. 2019;9(21):4528. doi: 10.3390/app9214528.

13. Jawaid P, Rehman MU, Zhao QL, Takeda K, Ishikawa K, Hori M, Shimizu T, Kondo T. Helium-based cold atmospheric plasma-induced reactive oxygen species-mediated apoptotic pathway attenuated by platinum nanoparticles. J Cell Mol Med. 2016;20(9):1737-48. doi: 10.1111/jcmm.12880.

14. Fridman G, Peddinghaus M, Balasubramanian M, Ayan H, Fridman A, Gutsol A, Brooks A. Blood coagulation and living tissue sterilization by floating-electrode dielectric barrier discharge in air. Plasma Chem Plasma Process. 2006;26:425-42 doi: 10.1007/s11090-006-9024-4.

15. Duske K, Wegner K, Donnert M, Kunert U, Podbielski A, Kreikemeyer B, Gerling T, Weltmann KD, Nebe B, Bader R. Comparative in vitro study of different atmospheric pressure plasma jets concerning their antimicrobial potential and cellular reaction. Plasma Process Polym. 2015;12(10):1050-60. doi: 10.1002/ppap.201400176.

16. Winter T, Bernhardt J, Winter J, Mäder U, Schlüter R, Weltmann KD, Hecker M, Kusch H. Common versus noble Bacillus subtilis differentially responds to air and argon gas plasma. Proteomics. 2013;13(17):2608-21. doi: 10.1002/pmic.201200343.

17. Chandana L, Sangeetha CJ, Shashidhar T, Subrahmanyam Ch. Non-thermal atmospheric pressure plasma jet for the bacterial inactivation in an aqueous medium. Sci Total Environ. 2018;640-1:493500. doi: 10.1016/j.scitotenv.2018.05.342.

18. Wende K, Williams P, Dalluge J, Gaens WV, Aboubakr H, Bischof J, von Woedtke T, Goyal SM, Weltmann KD, Bogaerts A, Masur K, Bruggeman PJ. Identification of the biologically active liquid chemistry induced by a nonthermal atmospheric pressure plasma jet. Biointerphases. 2015;10(2):029518. doi: 10.1116/1.4919710.

19. Kong MG, Kroesen G, Morfill G, Nosenko T, Shimizu T, van Dijk J, Zimmermann JL. Plasma medicine: An introductory review. New J Phys. 2009;11:115012. doi: 10.1088/1367-2630/11/11/115012.

20. Aboubakr HA, Mor SK, Higgins L, Armien A, Youssef MM, Bruggeman PJ, Goyal SM. Cold argon-oxygen plasma species oxidize and disintegrate capsid protein of feline calicivirus. PLoS One. 2018;13(3):e0194618. doi: 10.1371/journal.pone.0194618.

21. Martusevich AK, Galka AG, Karuzin KA, Tuzhilkin AN, Malinovskaya SL. Cold helium plasma as a modifier of free radical processes in the blood: In vitro study. AIMS Biophys. 2021;8(1):34-40. doi: 10.3934/biophy.2021002.

22. Martusevich AK, Kovaleva LK, Davyduk AV. Nitric oxide modulation of the crystallogenic properties of a biological fluid. Biophysics. 2016:61(2):291-6. doi: 10.1134/s0006350916020081.

23. Martusevich AK, Peretyagin SP. Modification of blood plasma crystallogenesis with nitrogen oxide processing. Biophysics. 2013;58(5):816-9. doi: 10.1134/s0006350913060134.

24. Rapis EG. Self-organization and supramolecular chemistry of protein films from the nano-to the macroscale. Tech Phys. 2004;49:494-8. doi: 10.1134/1.1736921.

25. Stoffels E, Sakiyama Y, Graves DB. Cold atmospheric plasma: Charged species and their interactions with cells and tissues. IEEE Trans Plasma Sci. 2008;36:1441-57. doi: 10.1109/tps.2008.2001084.

26. Martusevich AK, Galka AG, Golygina ES. Modifying the blood's physical and chemical parameters using cold helium plasma: In vitro study. Plasma Med. 2020;10(2):113-22. doi: 10.1615/ PlasmaMed.2020036212.

27. Ikehara Y, Sakakita H, Shimizu N, Ikehara S, Nakanish H. Formation of membrane-like structures in clotted blood by mild plasma treatment during hemostasis. J Photopol Sci Technol. 2013;26:555-7. doi: 10.2494/photopolymer.26.555.

Volume 11, Issue 1, 2021 
